\title{
Ellipsométrie spectroscopique de composés à base de nitrures.
}

\author{
M. Mihailovic, S. Colard, L. Siozade, J. Leymarie, P. Disseix, \\ A. Vasson et N. Antoine-Vincent \\ Laboratoire des Sciences et Matériaux pour l'Électronique et d'Automatique, \\ UMR CNRS/UBP 6602, Université Blaise Pascal Clermont-Ferrand II, \\ 24 avenue des Landais, F-63177 AUBIERE CEDEX, FRANCE \\ Martine.MIHAILOVIC@lasmea.univ-bpclermont.fr
}

\begin{abstract}
Résumé. Sur des films épais de GaN hexagonal, des mesures d'ellipsométrie spectroscopique et de réflectivité ont été réalisées à $300 \mathrm{~K}$ dans la gamme de longueurs d'onde 300 à $600 \mathrm{~nm}$. Elles ont permis de déterminer l'indice complexe de GaN en fonction de la longueur d'onde. Les spectres ellipsométriques nécessaires à l'étude optique des couches d'AlN et des couches tampons qui permettent leur croissance sur substrat de silicium sont aussi présentés.
\end{abstract}

Mots clés. Semiconducteurs, propriétés optiques, fonction diélectrique.

\section{INTRODUCTION}

Les composés du type $(\mathrm{Al}, \mathrm{Ga}) \mathrm{N}$ jouent un rôle de plus en plus important dans le domaine de l'optoélectronique depuis environ dix ans [1]. Un moyen d'améliorer les performances des diodes électroluminescentes et des photodétecteurs est d'utiliser des miroirs de Bragg et des cavités optiques. Ces structures sont formées d'empilements périodiques de fines couches de semiconducteurs. L'optimisation du nombre de périodes et du choix des matériaux permet de parfaitement contrôler l'évolution du champ électrique d'une onde lumineuse interagissant avec ces structures [2]. Pour cela, il est nécessaire de mesurer les indices optiques des composés de la famille $(\mathrm{Al}, \mathrm{Ga}) \mathrm{N}$.

\section{INFORMATIONS EXPÉRIMENTALES}

Les échantillons ont été réalisés au Centre de Recherche sur l'Hétéro-Épitaxie et ses Applications de Valbonne. Les détails de la croissance de GaN hexagonal sont présentés dans la référence [3] et d'AlN référence [4]. Les mesures ellipsométriques ont été obtenues sur un ellipsomètre spectroscopique à analyseur tournant pour deux angles d'incidence $72^{\circ}$ et $75^{\circ}$ et par pas de $2 \mathrm{~nm}$. Les mesures de réflectivité ont été faites en incidence normale.

\section{INDICE COMPLEXE DE GaN}

L'analyse des spectres ellipsométriques réalisés sur $\mathrm{GaN}$ hexagonal a été effectuée en deux temps :

- l'étude de la zone de transparence située à des longueurs d'onde supérieures à celle de la bande interdite permet de déterminer l'épaisseur du film de $\mathrm{GaN}(4,1 \mu \mathrm{m})$ et les paramètres de la loi de Sellmeier modélisant l'indice réel en fonction de la longueur d'onde :

$$
n=\sqrt{a+\left(b \lambda^{2} /\left(\lambda^{2}-c^{2}\right)\right),}
$$

avec $a=4,59 \pm 0,01, b=0,775 \pm 0,007$ et $c=303,5 \pm 0,4 \mathrm{~nm}$. 


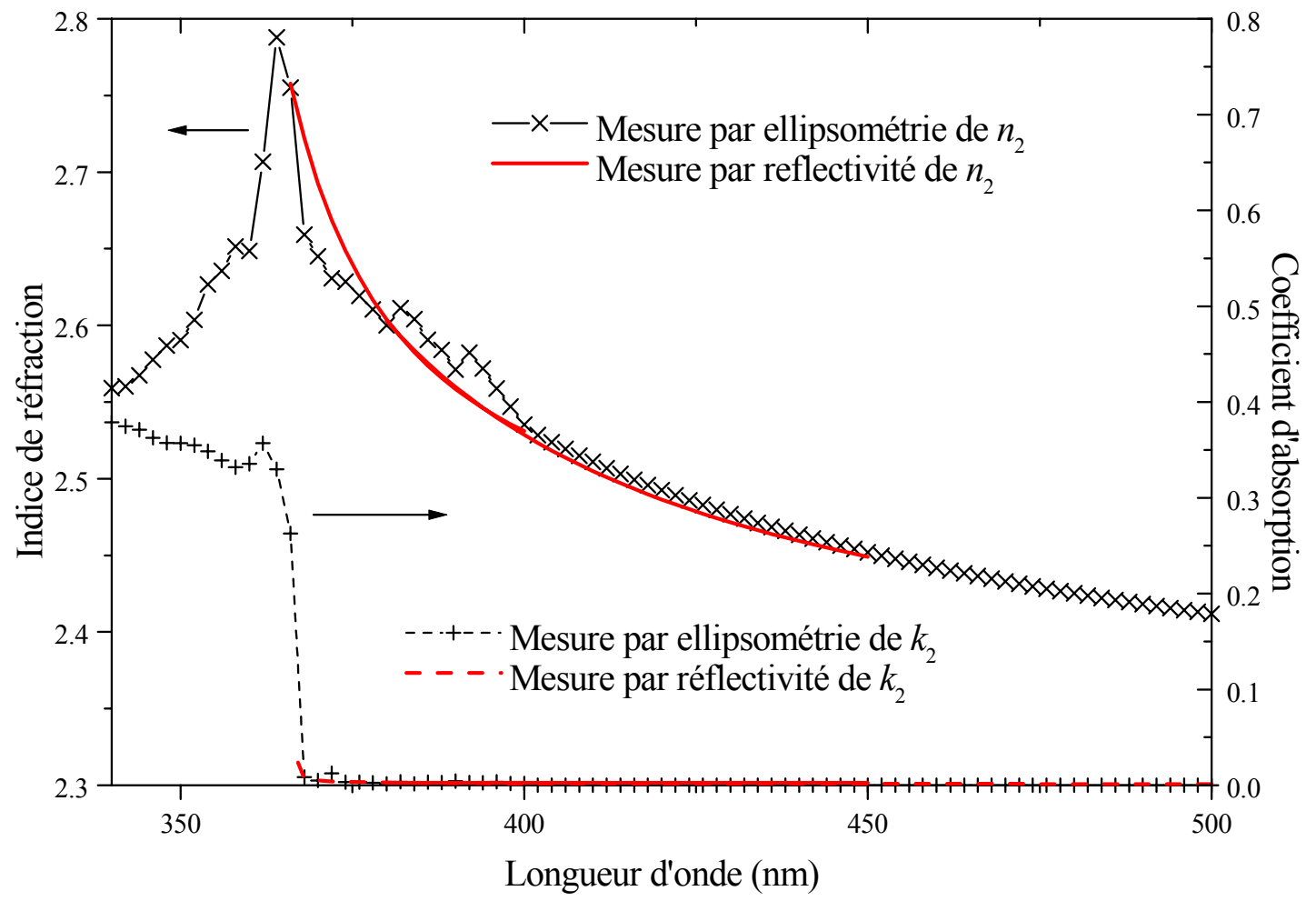

FIG. 1. Parties réelle et imaginaire de l'indice de réfraction de GaN hexagonal à $300 \mathrm{~K}$.

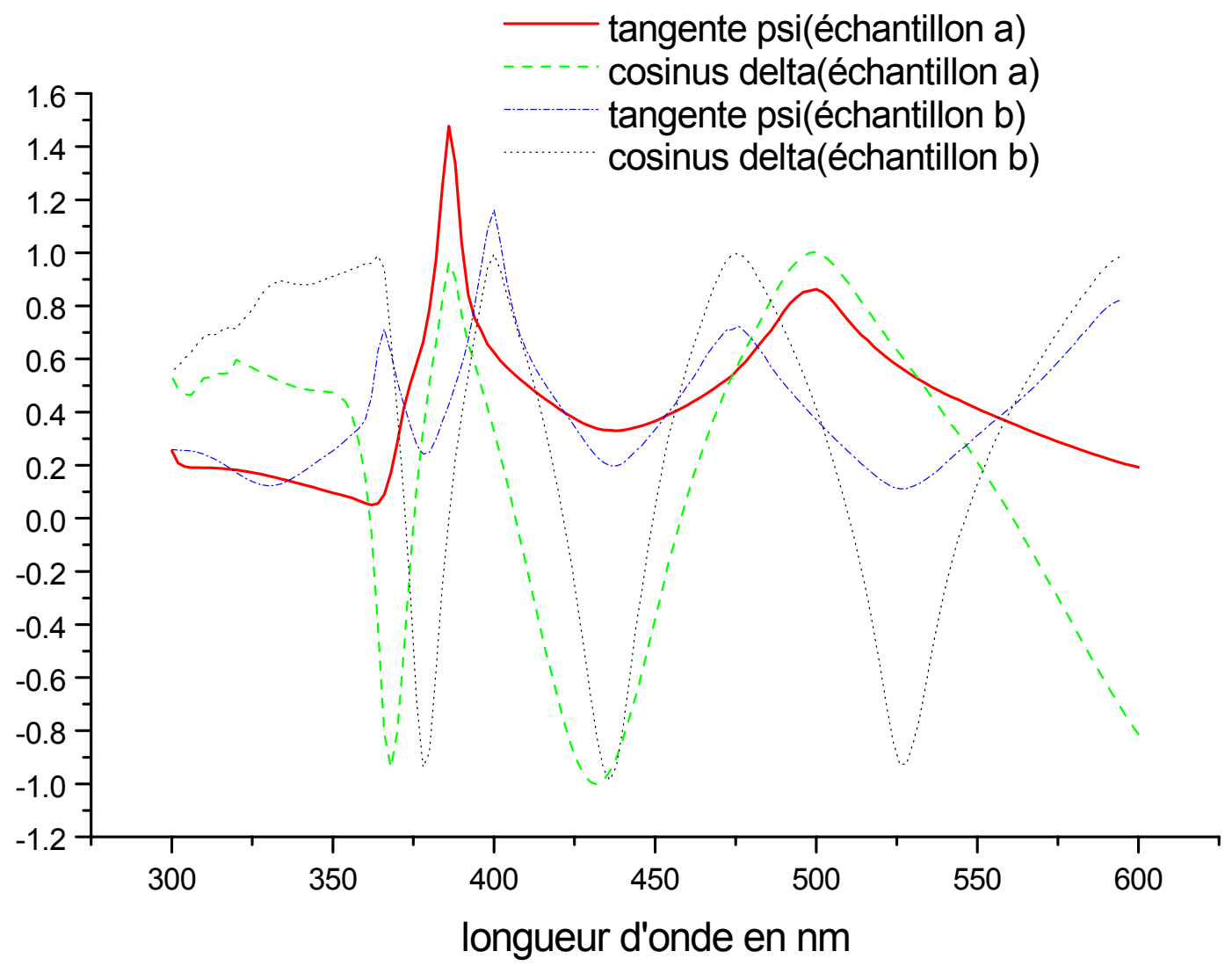

FIG. 2. Spectres ellipsométriques à l'incidence de $72^{\circ}$ et à $300 \mathrm{~K}$ d'une couche d'AlN sur couche tampon et substrat $\mathrm{Si}(\mathrm{b})$ et de la couche tampon sur substrat $\mathrm{Si}$ seule (a). 
Les résultats obtenus ont permis de calculer le spectre de réflectivité à $300 \mathrm{~K}$ en parfait accord avec l'expérience [ $\underline{3}]$;

- la connaissance de l'épaisseur du film de GaN autorise la détermination des parties réelle et imaginaire de l'indice longueur d'onde par longueur d'onde en deçà de la bande interdite. Les résultats sont présentés sur la figure 1.

\section{4. ÉTUDE PRÉLIMINAIRE DES ÉCHANTILLONS D'AIN}

La croissance d'une couche épaisse d'AIN nécessaire à la détermination de l'indice de ce matériau est assez difficile en raison de l'absence de substrat dont le paramètre de maille et le coefficient de dilatation soient voisins de ceux d'AlN. Il est donc nécessaire pour une bonne qualité cristalline d'interposer une couche tampon entre le substrat $\mathrm{Si}(111)$ et la couche d'AlN. Les spectres ellipsométriques mesurés à l'incidence de $72^{\circ}$ de la couche tampon seule sur le substrat et de la couche tampon sur substrat surmontée de la couche d'AlN sont présentés figure 2. L'analyse pour la détermination de l'indice d'AlN est en cours.

\section{CONCLUSION}

La connaissance des indices complexes est indispensable au design de structures comme les miroirs de Bragg et les microcavités. L'ellipsométrie est une méthode d'analyse optique qui donne accès aux épaisseurs de couches et/ou aux indices complexes des matériaux à travers un modèle pour interpréter la réponse d'un échantillon. Sur des couches de GaN dont la qualité cristalline est bien maîtrisée, c'est une méthode très bien adaptée à la détermination des paramètres optiques dont les résultats sont parfaitement corroborés par ceux de réflectivité et éventuellement complétés par des mesures d'absorption optique. Même dans le cas de structures multicouches plus compliquées comme celles étudiées pour AlN, l'ellipsométrie reste sans doute aussi la meilleure méthode pour obtenir les indices d'un matériau.

\section{RÉFÉRENCES}

[1] S. Nakamura et G. Fasol, The Blue Laser Diode, Springer-Verlag, Berlin (1997).

[2] N. Antoine-Vincent, F. Natali, P. Disseix, M. Mihailovic, A. Vasson, J. Leymarie, F. Semond, M. Leroux et J. Massies, Modeling and characterization of $\mathrm{GaN}$ microcavities, International Workshop on the Physics of Light-Matter Coupling in Nitrides, (Rome 26-29 sept. 2001), Phys. Stat. Sol. (a) 190 (2002) 187-192.

[3] L. Siozade, S. Colard, M. Mihailovic, J. Leymarie, A. Vasson, N. Grandjean, M. Leroux et J. Massies, Temperature dependence of optical properties of h-GaN films studied by reflectivity and ellipsometry, Jap. J. of Appl. Phys. 39 (2000) 20.

[4] F. Semond, N. Antoine-Vincent, N. Schnell, G. Malpuech, M. Leroux, J. Massies, P. Disseix, J. Leymarie et A. Vasson, Growth by molecular beam epitaxy and optical properties of a ten-period AlGaN/AlN Distributed Bragg Reflector on (111)Si, Phys. Stat. Sol. (a) 183 (2001) 163-167. 